\title{
ARTí́culo
}

\section{Consumo de oxígeno del pargo flamenco Lutjanus guttatus (Perciformes: Lutjanidae) durante su cultivo larval}

\author{
Oxygen consumption rates of spotted rose snapper Lutjanus guttatus \\ (Perciformes: Lutjanidae) during larval development
M. Isabel Abdo-de la Parra',2, M. Celene León-López ${ }^{3}$, L. Estela
Rodríguez-Ibarra ${ }^{1}$, Gustavo A. Rodríguez-Montes de $\mathbf{O c a}^{3}$, Gabriela Velasco-Blanco ${ }^{1}$ y José Cristóbal Román-Reyes $^{3}$

\begin{abstract}
${ }^{1}$ Centro de Investigación en Alimentación y Desarrollo, A.C., Av. Sábalo-Cerritos S/N, CP 82100, Mazatlán, Sinaloa, México ${ }^{2}$ Posgrado de Ciencias Agropecuarias, Colegio de Ciencias Agropecuarias, Universidad Autónoma de Sinaloa, Km. 17,5 Carretera Culiacán-Dorado, CP 8000 Culiacán, Sinaloa, México

${ }^{3}$ Laboratorio de Reproducción y Cultivo de Peces, Facultad de Ciencias del Mar, Universidad Autónoma de Sinaloa, Paseo Claussen s/n, C.P. 82000, Mazatlán, Sinaloa, México. jocrore@yahoo.com.mx
\end{abstract}

\begin{abstract}
Oxygen consumption of different larval stages of the spotted rose snapper Lutjanus guttatus were evaluated to help design food strategies for its culture. Oxygen consumption was determined using closed respirometers at a constant temperature of $30^{\circ} \mathrm{C}$ in a thermoregulated bath. Six replicates and 2 control for each treatment were used. Larval stages were evaluated from 2 to 35 days after hatching (DDE). Dissolved oxygen was measured using a fiber-optic microsensor connected to an oximeter. Respiration was expressed in microliters of oxygen per milligram of dry biomass per hour $\left(\mu \mathrm{LO}_{2} \mathrm{mg}^{-1} \mathrm{Bs} \mathrm{h}^{-1}\right)$. Results indicate that oxygen consumption rate $(R)$ decreased with the age of the larvae. Higher oxygen consumption was observed in the first 5 DDE which coincides with the onset of exogenous feeding. At 14, 19 and 30 DDE respiration rates showed slight increases and appear to be associated with morphological changes of the larvae (preflexion, flexion and postflexion, respectively). The values of the exponents of the potential allometric relationship between the specific biomass respiration rate and for each individual, based on the dry biomass of the spotted rose snapper were -0.172 and 0.834 respectively. The data obtained are important to estimate oxygen demand during the larval rearing of the spotted rose snapper, and combined with nitrogen losses can be used to estimate feeding requirements based on energy losses.
\end{abstract}

Key words: Respiratory rates, spotted rose snapper, larvae

Resumen.- Se evaluó el consumo de oxígeno de los diferentes estadios larvales del pargo flamenco Lutjanus guttatus para contribuir al diseño de estrategias de alimentación para su cultivo. El consumo de oxígeno se determinó utilizando respirómetros cerrados a una temperatura constante de $30^{\circ} \mathrm{C}$ en una bañera termorregulada. Se utilizaron 6 repeticiones y 2 testigos por cada tratamiento. Se evaluaron los estadios larvarios desde el día 2 hasta los 35 días después de la eclosión (DDE). El oxígeno disuelto se cuantificó utilizando un microsensor de fibra óptica conectado a un oxímetro. La respiración, fue expresada en microlitros de oxígeno por miligramo de biomasa seca por hora $\left(\mu \mathrm{LO}_{2} \mathrm{mg}^{-1} \mathrm{Bs} \mathrm{h}^{-1}\right)$. Los resultados indicaron que la tasa de consumo de oxígeno ( $\mathrm{R}$ ) disminuyó con la edad de las larvas. El mayor consumo de oxígeno fue en los primeros 5 DDE lo cual coincide con el inicio de la alimentación exógena. A los 14, 19 y 30 DDE las tasas respiratorias presentaron ligeros incrementos, y parecen estar asociados a cambios morfológicos de las larvas (preflexión, flexión y posflexión, respectivamente). Los valores de los exponentes de la relación potencial alómetrica entre la tasa respiratoria biomasa-específica y por individuo, en función de la biomasa seca del pargo flamenco, fueron de $-0,172$ y 0,834, respectivamente. Los datos obtenidos son importantes para estimar las demandas de oxígeno durante el cultivo larvario del pargo flamenco, y combinados con las pérdidas de nitrógeno, pueden ser usados para estimar los requerimientos de alimentación en base a las pérdidas energéticas.

Palabras clave: Tasa respiratoria, pargo flamenco, larvas 


\section{INTRODUCCIÓN}

El pargo flamenco Lutjanus guttatus (Steindachner, 1869) se distribuye en el Pacífico oriental, desde México hasta Perú (Fischer et al. 1995). Es una especie demersal que habita arrecifes costeros de hasta $30 \mathrm{~m}$ de profundidad, generalmente solitaria o en pequeños cardúmenes y ocasionalmente forma grandes cardúmenes (Allen 1995). Presenta alta demanda en los mercados de algunos países de Latinoamérica; por lo que a partir de la década pasada, en el Pacífico mexicano los pescadores extraen juveniles silvestres para engordarlo en jaulas (Avilés-Quevedo et al. 2008). Las investigaciones sobre su reproducción artificial se iniciaron hace varios años en algunos países como Colombia, Costa Rica, Panamá y Ecuador (BozaAbarca et al. 2008, Alvarez-Lajonchère \& Puello-Cruz 2011). En México, las investigaciones se iniciaron en el Centro de Investigación en Alimentación y Desarrollo, Unidad Mazatlán (CIAD), desde el año 2000 (García-Ortega et al. 2005). En la actualidad se ha logrado la producción masiva de juveniles y se completó en condiciones de cultivo el ciclo generacional (IbarraCastro et al. 2012); sin embargo, todos los trabajos relacionados al respecto han mostrado que durante los primeros estadios de vida se presenta una alta mortalidad, con una supervivencia al final del cultivo larvario entre 1 a 12\% (GarcíaOrtega et al. 2005, Boza-Abarca et al. 2008, Abdo-de la Parra et al. 2010, Alvarez-Lajonchère et al. 2012). Las altas mortalidades que se han presentado en la larvicultura del pargo flamenco, ha sido uno de los principales problemas en el desarrollo e implementación de la biotecnología del cultivo a nivel comercial. En la actualidad no se han realizado trabajos para determinar la relación que existe entre el metabolismo y consumo de oxígeno de las larvas de pargo flamenco y la mortalidad durante los primeros estadios de vida, lo cual optimizaría las técnicas de cultivo mejorandola salud y calidad de los juveniles de pargo producidos en cautiverio. La energía necesaria para el metabolismo de rutina se deriva de la dieta, principalmente de los aminoácidos y lípidos. En general, la energía consumida es directamente proporcional al oxígeno consumido en cada célula (Miyashima et al. 2012). La tasa metabólica es la velocidad a la que un organismo utiliza la energía disponible y se estima como la tasa de liberación de calor del organismo, que se obtiene midiendo la liberación de calor (calorimetría directa) o la tasa de consumo de oxígeno (calorimetría indirecta) (Calderer-Reig 2001). Por lo tanto, el oxígeno consumido por las larvas de peces se utiliza como un indicador fisiológico muy importante del metabolismo (Rombough 1988). Varios autores han demostrado que la tasa de consumo de oxígeno en las larvas de peces varía de acuerdo al estadio de desarrollo (Oozeki \& Hirano 1994); sin embargo, la relación entre la tasa metabólica y la masa corporal depende de la especie (Pelster 2008); por lo tanto, es importante conocer el consumo de oxígeno de un organismo, ya que es un buen indicador del estado metabólico general del pez y es una medida sensible del gasto diario de energía, que necesita el organismo para mantener sus funciones vitales a través de un metabolismo aeróbico. El objetivo del presente trabajo fue determinar la tasa de consumo de oxígeno de los diferentes estadios larvales del pargo flamenco, para contribuir al diseño de estrategias de alimentación que cubran en tiempo y forma sus requerimientos energéticos, mejorar las condiciones del cultivo y disminuir, en consecuencia, las altas mortalidades presentadas en esta etapa.

\section{Material Y MÉTOdos}

\section{INCUBACIÓN DE HUEVOS Y LARVICULTURA}

Los huevos viables de pargo flamenco fueron obtenidos del lote de reproductores de la planta piloto para la producción de juveniles de peces marinos del Centro de Investigación en Alimentación y Desarrollo, Unidad Mazatlán (CIAD), los huevos viables son flotantes, esféricos, con el vitelo homogéneo y transparente, con un diámetro aproximado de 724-728 $\mu \mathrm{m}$ y una sola gota de aceite con 121-123 $\mu \mathrm{m}$ de diámetro. Se incubaron en 5 jarras tipo McDonald de $6 \mathrm{~L}$ de capacidad (2000 huevos $\left.\mathrm{L}^{-1}\right)$, con flujo de aire $\left(0,5 \mathrm{~L} \mathrm{~min}^{-1}\right)$ y a $29^{\circ} \mathrm{C}$. Las larvas recién eclosionadas (1,8 a $2 \mathrm{~mm}$ de longitud total) fueron transferidas a 2 tanques circulares de fibra de vidrio de 3.000 L donde se llevó a cabo la larvicultura, a una temperatura media del agua de $30 \pm 1,0^{\circ} \mathrm{C}$ y salinidad de 35 . El cultivo larvario se realizó mediante el protocolo propuesto por Abdo-de la Parra et al. (2010); a partir del día 1 a 13 días después de la eclosión (DDE), se añade a los tanques de cultivo una mezcla de 500.000 células $\mathrm{ml}^{-1}$ de Nannochloropsis oculata y 50.000 células $\mathrm{ml}^{-1}$ de Isochrysis sp. Desde el día 2 hasta el 6DDE se entregó en cada tanque 10 rotíferos $\mathrm{ml}^{-1}$ (Brachionus rotundiformis), previamente enriquecidos durante $3 \mathrm{~h}$ con microalgas (Nannochloropsis oculata), incrementando la ración a partir del 7 a 15 DDE a 20 rotíferos $\mathrm{ml}^{-1}$. Además, a partir de 2 DDE se añadió una mezcla de copepoditos y adultos de Tisbe monozota y Pseudodiaptomus euryhalinus, a una concentración de 0,5 presas $\mathrm{ml}^{-1}$, que se mantuvo constante hasta 15 DDE. Del 16 al 35 DDE, la cantidad de rotíferos se redujo a 10 presas $\cdot \mathrm{ml}^{-1}$ y se añadieron 0,5 nauplios de Artemia $\cdot \mathrm{ml}^{-1}$, que se incrementaron gradualmente hasta 4 nauplios $\cdot \mathrm{ml}^{-1}$.

\section{Cámaras Respirométricas Y DETERMINACIÓN DEL} CONSUMO DE OXÍGENO

Para los experimentos se utilizaron los estadios larvarios del 2, 4, 5, 8, 11, 14, 16, 19, 23, 30 y 35 DDE. Se emplearon cámaras respirométricas cerradas con volumen de $310 \mathrm{ml}$ (botellas BOD 
transparentes), con 6 repeticiones por estadio, colocando entre 5 y 50 larvas por respirómetro dependiendo de la etapa larvaria y 2 controles sin organismos. Las larvas recién alimentadas fueron colocadas en los respirómetros y se llenaron a su máxima capacidad con agua filtrada a $1 \mu \mathrm{m}$ y mantenidos a temperatura constante de $30^{\circ} \mathrm{C}$, temperatura adecuada para el cultivo de esta especie (Álvarez-Lajonchere \& Puello-Cruz 2011), en un baño termorregulado con un calentador de titanio de $1.500 \mathrm{~W}$. Los organismos se aclimataron antes de iniciar los experimentos, por al menos $1 \mathrm{~h}$ en los respirómetros, bajo luz natural. Al terminar el periodo de aclimatación se tomó la lectura inicial del oxígeno disuelto, procurando que la concentración no fuera menor de los $5 \mathrm{mg} \mathrm{L}^{-1}$

Para determinar la concentración de oxígeno disuelto se colectaron $3 \mathrm{ml}$ de muestra de cada respirómetro mediante una jeringa de $3 \mathrm{ml}$ de capacidad, parte de la cual se inyectó en una cámara de vidrio hermética de $100 \mu$ l. A la cámara se le adaptó un microsensor de fibra óptica (PreSens, GMBH, Alemania), el cual a su vez se conectó a un oxímetro MICROX TX3, que transmitió los datos de oxígeno disuelto $\left(\mathrm{mg} \mathrm{O}_{2} \mathrm{~L}^{-1}\right)$ al disco duro de una computadora a través de una interfase en serie capaz de transmitir mediciones de oxígeno a intervalos de un segundo. La ventaja de esta técnica es que permite mediciones rápidas de oxígeno disuelto, tiene alta sensibilidad, no consume oxígeno durante las mediciones y no es afectada por la salinidad (Klimant et al. 1995). El microsensor fue calibrado a la temperatura y salinidad experimental, en agua aireada al $100 \%$ de saturación y una solución de $0,5 \%$ de $\mathrm{NaSO}_{3}$ con $0 \%$ de oxígeno como estándar.

El consumo de oxígeno fue expresado en unidades de $\mu \mathrm{g}$ de $\mathrm{O}_{2} \mathrm{mg}^{-1}$ de Biomasa Seca (Bs) $\mathrm{h}^{-1}$ o de $\mu \mathrm{g}$ de $\mathrm{O}_{2}$ larva $\mathrm{h}^{-1}$, los cuales se calcularon con base a la ecuación: $R=\left(\Delta \mathrm{O}_{2} * \mathrm{VH}_{2} \mathrm{O}\right) /$ (Wo* $\Delta \mathrm{t}$ ), Donde $\Delta \mathrm{O}_{2}$ es la diferencia de la concentración de oxígeno entre el respirómetro control y los respirómetros experimentales al final de cada periodo, $\mathrm{VH}_{2} \mathrm{O}$ es el volumen de agua en los respirómetros en $\mathrm{ml}$, Wo es la biomasa seca en mg o el número de larvas y $\Delta$ t es el tiempo trancurrido en horas. El tiempo total de experimentación fue de $2 \mathrm{~h}$ con muestreos consecutivos cada media hora, ya que Ameer \& Kutty (1972) indicaron que es el tiempo máximo para no permitir concentraciones de oxígeno menores a $2 \mathrm{mg} \mathrm{L}^{-1}$ y evitar el estrés de los organismos. En cada fase de experimentación se midió la longitud total (LT) de las larvas mediante un vernier digital de 0,01 mm de precisión y se determinó el peso seco (Bs), colocando entre 10 a 50 larvas en papel secante para secarlas en una estufa a $60^{\circ} \mathrm{C}$ durante $8 \mathrm{~h}$ y posteriormente se pesaron en una balanza semimicro Denver Instrument PI-225D con una precisión de 0,01 mg.

\section{Análisis estadístico}

Los resultados fueron analizados mediante un análisis de varianza (ANDEVA) no paramétrico de Kruskal-Wallis $(P<0,05)$, ya que se rechazaron los supuestos de normalidad y homogeneidad de varianzas en los datos (Zar 1999). Cuando se presentaron diferencias significativas se realizaron pruebas de comparaciones múltiples de media no paramétricas de Dunn. Se realizaron regresiones lineales y no lineales para examinar las relaciones entre la tasa de consumo de oxígeno, la edad y la biomasa seca de las larvas.

\section{Resultados}

\section{Biomasa SECA (Bs) y LONGITUd TOTAL (LT)}

Las larvas de 2 DDE presentaron una Bs de 0,01 $\pm 0,001 \mathrm{mg}$ hasta alcanzar 58,48 $\pm 3,14 \mathrm{mg}$ el $35 \mathrm{DDE}$. A los 2 DDE las larvas midieron alrededor de 2,08 $\pm 0,14 \mathrm{~mm}$ de LT hasta alcanzar 26,63 \pm 3,03 $\mathrm{mm}$ de LT a los 35 DDE (Tabla 1).

Tasa de CONSUMo de OXígeno BIOMASA-ESPECífica $\left(\mu \mathrm{LO}_{2} \mathrm{MG}^{-1} \mathrm{BS} \mathrm{H}^{-1}\right)$ Y POR LARVA $\left(\mu \mathrm{LO}_{2}\right.$ LARVA H$\left.^{-1}\right)$ EN RELACIÓN A LA EDAD DE LAS LARVAS DE PARGO FLAMENCO

Conforme aumenta la edad de las larvas, se observó que el consumo de oxígeno disminuye significativamente (KruskalWallis, $\mathrm{H}=48,38, \mathrm{gl}=10, P<0,05$ ) (Tabla 1$)$. La relación entre el consumo de oxígeno-biomasa específica y la edad de las larvas se muestra en la Figura 1 y se pueden observar ligeros incrementos en el consumo de oxígeno en los 14, 19 y 30 DDE.

La tasa de consumo de oxígeno por larva $\left(\mu \mathrm{LO}_{2}\right.$ larva $\left.^{-1}\right)$ se incrementó exponencialmente con la edad de las larvas de acuerdo a la ecuación $\mathrm{R}=0,366 \cdot \mathrm{e}^{0,187 \cdot \text { dde }}\left(\mathrm{r}^{2}=0,9897, P<0,05\right)$ (Fig. 2). El análisis de varianza detectó diferencias significativas el consumo de oxígeno por larva y la edad (Kruskal-Wallis, $\mathrm{H}=52,68, \mathrm{gl}=10, P<0,05)$, presentándose los valores más bajos entre las larvas de 2 días hasta $11 \mathrm{DDE}$; mientras que entre en los 14 a 19 DDE, la tasa respiratoria por larva presentó incrementos importantes sin ser estadísticamente diferentes entre ellos $(P>0,05)$. Posteriormente, los valores del consumo de oxígeno se incrementaron significativamente de manera continua a los 23, 30 y 35 DDE (Tabla 1).

TASa ReSPIRATORIa BIOMASA-ESPECífica $\left(\boldsymbol{\mu} \mathrm{LO}_{2} \mathrm{MG}^{-1} \mathrm{Bs}\right.$ $\left.\mathrm{H}^{-1}\right)$ Y POR LARVA $\left(\mu \mathrm{LO}_{2}\right.$ LARVA $\left.{ }^{-1}\right)$ EN FUNCIÓN DE LA BIOMASA SECA (Bs) DE LAS LARVAS DE PARGo FlaMENCO.

La tasa de consumo de oxígeno biomasa-específica $\left(\mu \mathrm{LO}_{2}\right.$ $\mathrm{mg}^{-1} \mathrm{Bs} \mathrm{h}^{-1}$ ) disminuyó significativamente con el incremento en la biomasa seca (Bs). Los datos fueron transformados a logaritmos de base 10 para linealizar la relación. La ecuación de regresión $\log \mathrm{R}=0,842-0,172 * \log \mathrm{Bs}\left(\mathrm{r}^{2}=0,844, P<0,05\right)$ 


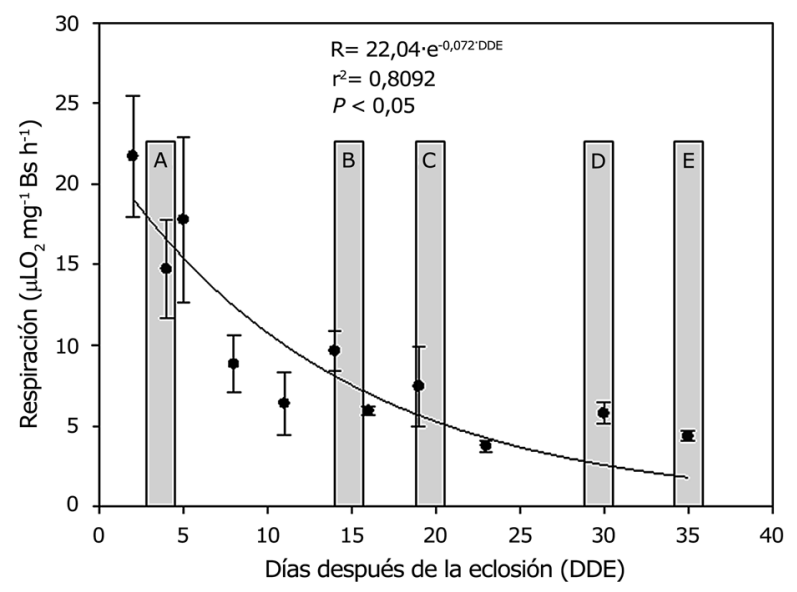

Figura 1. Tasa respiratoria $\left(\mu \mathrm{LO}_{2} \mathrm{mg}^{-1} \mathrm{Bsh} \mathrm{h}^{-1}\right)$ de larvas del pargo flamenco Lutjanus guttatus en relación con la edad (DDE). La barras señalan $A$ : inicio de la alimentación exógena, B: preflexión, C: flexión, D: posflexión y E: inicio del destete. Los puntos indican los promedios de la tasa respiratoria y las barras verticales representan \pm la desviación estándar /Spotted rose snapper Lutjanus guttatus larval respiratory rate $\left(\mu \mathrm{LO}_{2} \mathrm{mg}^{-1} \mathrm{Bs} \mathrm{h}^{-1}\right)$ in relation to age. A: Start of exogenous feeding, B: Preflexion, C: Flexion, D: Postflexion, E: Weaning. The points indicate the average respiratory rate and vertical bars represent \pm standard deviation

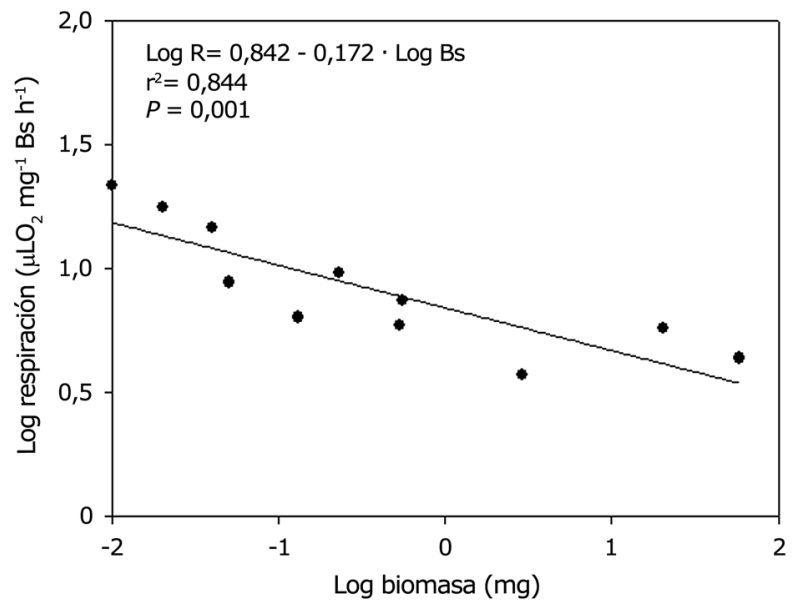

Figura 3. Gráfico log-log entre la tasa de respiración $\left(\mu \mathrm{LO}_{2} \mathrm{mg}^{-1} \mathrm{Bsh}^{-1}\right)$ del pargo flamenco Lutjanus guttatus con la biomasa seca en $\mathrm{mg}$ (Bs) / Log-log graph shows the relationship between respiration rate $\left(\mu \mathrm{LO}_{2}\right.$ $\mathrm{mg}^{-1} \mathrm{Bs} \mathrm{h}^{-1}$ ) with dry weight in $\mathrm{mg}$ (Bs) of the spotted rose snapper Lutjanus guttatus

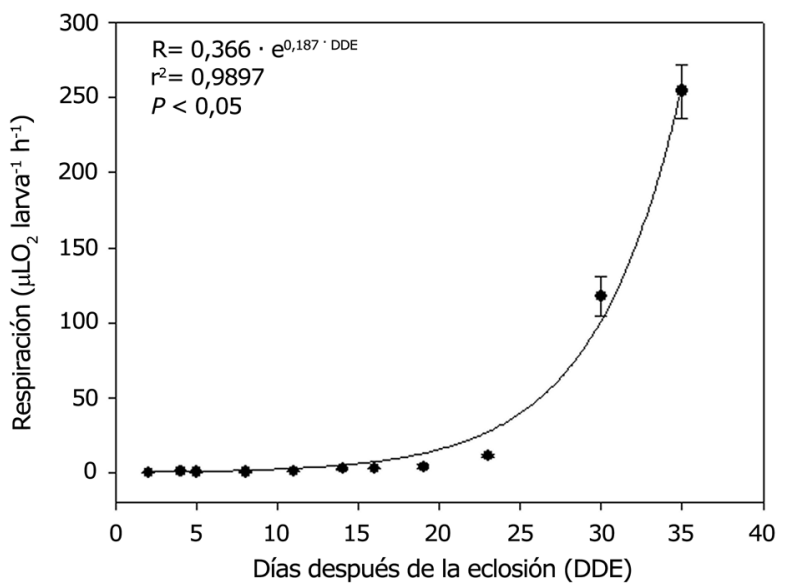

Figura 2. Tasa respiratoria por individuo $\left(\mu \mathrm{LO}_{2} \operatorname{larva}^{-1} \mathrm{~h}^{-1}\right)$ de larvas de pargo flamenco Lutjanus guttatus en relación con la edad (DDE). Los puntos indican los promedios de la tasa respiratoria y las barras verticales representan \pm la desviación estándar de la media / Spotted rose snapper Lutjanus guttatus individual larval respiratory rate $\left(\mu \mathrm{LO}_{2}\right.$ larva $\left.^{-1} \mathrm{~h}^{-1}\right)$ in relation to age (DDE). The dots indicate the average respiratory rate and vertical bars represent \pm standard deviation

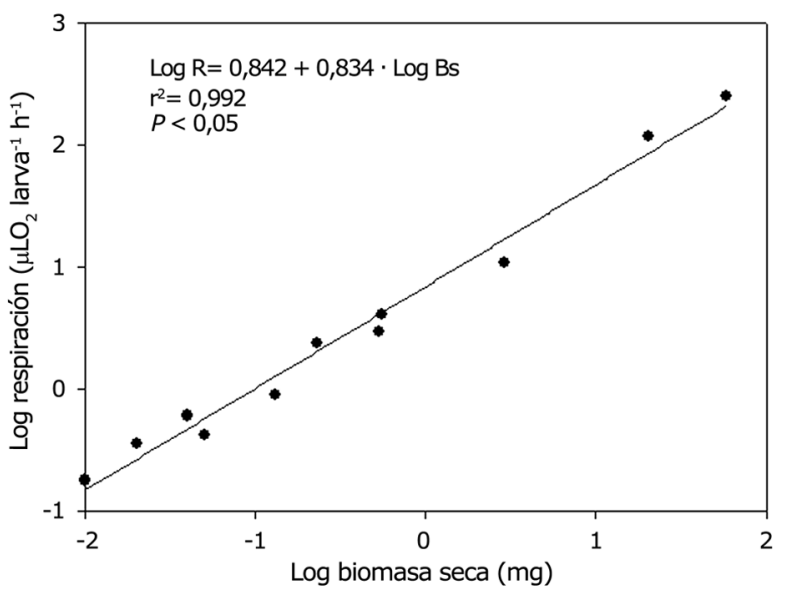

Figura 4. Gráfico log-log entre la tasa de respiración por larva $\left(\mu \mathrm{LO}_{2}\right.$ larva ${ }^{-1} h^{-1}$ ) del pargo flamenco Lutjanus guttatus con la biomasa seca en $\mathbf{m g}$ (Bs) / Log-log graph shows the relationship between larval respiration rate $\left(\mu \mathrm{LO}_{2}\right.$ larva $\left.^{-1} \mathrm{~h}^{-1}\right)$ with dry weight of the spotted rose snapper Lutjanus guttatus 
Tabla 1. Promedios \pm desviación estándar $(\bar{X} \pm D E$ ) de la biomasa seca, longitud total (LT) y tasa de consumo de oxígeno de larvas de Lutjanus guttatus hasta 35 días después de la eclosión (DDE). Los superíndices diferentes en la columna del consumo de oxígeno indican diferencias significativas ( $P<$ $\mathbf{0 , 0 5}$ / Mean \pm standard deviation ( $\bar{X} \pm S D$ ) dry biomass, total length $(T L)$ and rate of oxygen consumption of $L$. guttatus larvae up to 35 days after hatching (DDE). Different superscripts indicate significant differences $(P<0.05)$

\begin{tabular}{cccccc}
\hline $\begin{array}{c}\text { Días después } \\
\text { de la eclosión }\end{array}$ & $\begin{array}{c}\text { Número } \\
\text { de larvas }\end{array}$ & $\begin{array}{c}\text { Biomasa seca } \\
\left(\mathrm{mg} \mathrm{larva}^{-1}\right)\end{array}$ & $\begin{array}{c}\text { Longitud } \\
\text { total }(\mathrm{mm})\end{array}$ & $\begin{array}{c}\text { Consumo de } \\
\left.\text { oxígeno }^{-1} \mathrm{LO}_{2} \mathrm{mg}^{-1} \mathrm{~h}^{-1}\right)\end{array}$ & $\begin{array}{r}\text { Consumo de } \\
\mathrm{O}_{2} \text { por larva } \\
\left(\mu \mathrm{LO}_{2} \text { larva h}^{-1}\right)\end{array}$ \\
\hline 2 & 50 & $0,01 \pm 0,001$ & $2,08 \pm 0,14$ & $37,07 \pm 3,73^{\mathrm{c}}$ & $0,18 \pm 0,03^{\mathrm{a}}$ \\
4 & 50 & $0,04 \pm 0,001$ & $2,43 \pm 0,19$ & $22,16 \pm 5,90^{\mathrm{b}}$ & $0,61 \pm 0,13^{\mathrm{a}}$ \\
5 & 45 & $0,02 \pm 0,01$ & $2,63 \pm 0,16$ & $26,26 \pm 4,16^{\mathrm{bc}}$ & $0,36 \pm 0,1^{\mathrm{a}}$ \\
8 & 40 & $0,05 \pm 0,03$ & $3,31 \pm 0,18$ & $8,83 \pm 1,77^{\mathrm{a}}$ & $0,42 \pm 0,08^{\mathrm{a}}$ \\
11 & 30 & $0,13 \pm 0,06$ & $4,18 \pm 0,50$ & $6,37 \pm 1,93^{\mathrm{a}}$ & $0,91 \pm 0,28^{\mathrm{a}}$ \\
14 & 25 & $0,23 \pm 0,08$ & $5,26 \pm 0,74$ & $9,82 \pm 0,98^{\mathrm{a}}$ & $2,41 \pm 0,31^{\mathrm{b}}$ \\
16 & 25 & $0,53 \pm 0,05$ & $6,14 \pm 0,67$ & $5,92 \pm 0,26^{\mathrm{a}}$ & $2,96 \pm 0,13^{\mathrm{b}}$ \\
19 & 20 & $0,55 \pm 0,08$ & $8,24 \pm 0,83$ & $10,99 \pm 0,55^{\mathrm{a}}$ & $4,09 \pm 1,37^{\mathrm{b}}$ \\
23 & 15 & $2,92 \pm 0,73$ & $10,55 \pm 1,33$ & $3,72 \pm 0,36^{\mathrm{a}}$ & $10,87 \pm 1,04^{\mathrm{c}}$ \\
30 & 10 & $20,40 \pm 2,16$ & $17,10 \pm 3,74$ & $7,52 \pm 1,08^{\mathrm{a}}$ & $117,62 \pm 13,61^{\mathrm{d}}$ \\
35 & 5 & $58,48 \pm 3,14$ & $26,63 \pm 3,03$ & $4,35 \pm 0,31^{\mathrm{a}}$ & $254,41 \pm 17,84^{\mathrm{e}}$ \\
\hline
\end{tabular}

se muestra en la Figura 3. La ecuación logarítmica transformada a la versión potencial alométrica resulta en la ecuación $\mathrm{R}=$ $6,94 * \mathrm{Bs}^{-0,172}$.

Por otro lado, la tasa respiratoria por larva $\left(\mu \mathrm{LO}_{2} \operatorname{larva}^{-1} \mathrm{~h}^{-1}\right)$ aumentó significativamente conforme aumenta la Bs. La relación fue descrita por la ecuación logarítmica $\log \mathrm{R}=$ $0,842+0,834 * \log \mathrm{Bs}\left(\mathrm{r}^{2}=0,992, P<0,05\right)$ y la correspondiente versión alométrica es $\mathrm{R}=6,94 * \mathrm{Bs}^{0,834}$ (Fig. 4).

\section{Discusión}

El presente trabajo es el primer estudio reportado hasta la fecha que describe la tasa respiratoria del pargo flamenco Lutjanus guttatus durante el desarrollo larvario, desde el DDE 2 hasta los 35 DDE y proporciona información útil sobre el requerimiento metabólico de la especie, lo cual puede contribuir al avance de la biotecnología para su cultivo. Jobling (1994) señala que el consumo de oxígeno es uno de los perfiles fisiológicos más útiles para estimar los requerimientos nutricionales de los peces, ya que representa la tasa del metabolismo energético. En un amplio sentido, el término metabolismo, es la suma de todas las reacciones químicas que ocurren en un organismo y la medición del oxígeno consumido es un método usado comúnmente para determinar indirectamente la tasa metabólica de los organismos (De la Gándara 2003). En este aspecto, Winberg (1960) define 3 niveles de metabolismo: la tasa metabólica estándar, la cual es medida en peces mantenidos reposo y sin alimentar; la tasa metabólica de rutina, medida en peces sin alimentar pero que muestran nado espontaneo; la tasa metabólica activa, que se define como la tasa aeróbica máxima sostenible. En consecuencia, la tasa metabólica estándar representa la demanda energética mínima que un animal requiere para mantenerse con vida, pero que no está asociada con la natación, digestión y catabolismo (Benetti et al. 1995). Los valores en el consumo de oxígeno reportados en este estudio pudieran representar el metabolismo de rutina más la acción dinámica específica, puesto que los experimentos fueron realizados con organismos con contenido estomacal y confinadas en los respirómetros donde se observó nado continuo (aunque no se midió la velocidad de nado). Las mayores tasas de consumo de oxígeno $\left(\mu \mathrm{LO}_{2} \mathrm{mg}^{-1} \mathrm{Bs} \mathrm{h}^{-1}\right)$ se presentaron en los primeros 5 DDE, coincidiendo con la absorción del saco vitelino y con el inicio de la alimentación exógena y por consiguiente con el incremento en la actividad por la búsqueda del alimento, lo cual ha sido observado en otras especies de peces marinos como Pagrus pagrus (Aristizabal 2006), Paralichthys olivaceus, Pagrus major e Inimicus japonicus (Myrashima et al. 2012) donde se reporta un incremento en el consumo de oxígeno en los primeros estadios de vida, cuando se presente la apertura de la boca, el consumo del saco vitelino e inicia la alimentación exógena. Con base en sus resultados, Miyashima et al. (2012) hipotetizaron que las larvas de peces marinos requieren más energía durante el periodo comprendido entre la apertura de la boca y flexión de la notocorda y por consecuencia el porcentaje de mortalidad se eleva cuando la energía proporcionada es insuficiente. Por consiguiente, las altas tasas metabólicas determinadas en este estudio en los primeros días 
después de la eclosión, pueden contribuir a las elevadas mortalidades reportadas al inicio de la alimentación exógena de las larvas de pargo flamenco (Boza-Abarca et al. 2008, Abdo-de la Parra et al. 2010). Por otro lado, los valores observados de consumo de oxígeno entre los 8 y 35 DDE no parecen estar asociados a cambios morfológicos de las larvas de pargo; excepto a los 14, 19 y $30 \mathrm{DDE}$, donde se observaron incrementos (aunque no significativos) en las tasas respiratorias, los cuales coinciden con la preflexión, la flexión y posflexión del notocordio en las larvas. Boza-Abarca et al. (2008) y Abdo de la Parra \& Rodríguez-Ibarra (2011) mencionan que en las larvas de pargo flamenco la preflexión ocurre entre los 14 a 16 DDE, la flexión a los 19-20 DDE y la postflexión a los 27 DDE. Estos cambios en el consumo de oxígeno también fueron observados por Ishibashi et al. (2005) en los estadios tempranos de Pagrus major, quienes indicaron que alrededor de la etapa de flexión y postflexión las demandas metabólicas se incrementaron. Por otro lado, la disminución de la tasa de consumo de oxígeno biomasa-específica $\left(\mu \mathrm{LO}_{2} \mathrm{mg}^{-1} \mathrm{Bs} \mathrm{h}^{-1}\right)$ respecto a la edad de las larvas ha sido reportada para varias especies de peces, la cual disminuye con el incremento en la biomasa de los organismos (Djawad et al. 1995, Barrionuevo \& Burggren 1999, Pelster 2008, Barrionuevo et al. 2010), debido a que los peces con menor biomasa consumen más oxígeno por unidad de masa que sus congéneres con mayor biomasa (Jobling 1994). La reducción de la tasa de consumo de oxígeno biomasa-específica con el desarrollo larval, es el resultado del aumento de la biomasa de tejidos con tasas metabólicas menores (músculo blanco), relativo a los tejidos con tasas metabólicas mayores (ciego pilórico, cerebro, intestinos), los cuales son cada vez menos pesados en proporción a todo el cuerpo, conforme el organismo crece (Oikawa et al. 1992, Oozeki \& Hirano 1994). Por el contrario, la tasa respiratoria del pargo flamenco por larva $\left(\mu \mathrm{LO}_{2}\right.$ larva $^{-1}$ $\left.\mathrm{h}^{-1}\right)$ se incrementó exponencialmente en relación con la edad de las larvas $\left(\mathrm{R}=0,336 * \mathrm{e}^{0,187 \mathrm{DDE}}\right)$. Estos resultados confirman la relación exponencial existente entre la tasa de consumo de oxígeno por individuo y la edad de las larvas reportadas para otras especies de peces marinos (Houde \& Sckeckter 1983, Houde \& Zastrow 1993, Oozeki \& Hirano 1994, Torres et al. 1996, Gisbert et al. 2002, Idrisi et al. 2002). El incremento exponencial es atribuido al rápido crecimiento de la superficie de intercambio de gases, siendo la superficie corporal el sitio principal para el intercambio de gases en los estados más tempranos de desarrollo, antes de la formación de las branquias (Rombough 1988). El incremento exponencial en el consumo de oxígeno de las larvas de pargo flamenco a partir de los 14 DDE, se asume como una respuesta a una rápida formación de las lamelas branquiales (sitio de mayor intercambio de gases), lo cual sucede en la mayoría de larvas de peces entre los 8 y 12
DDE (Iwai \& Hughes 1977, Oozeki \& Hirano 1994). El desarrollo de arcos y filamentos branquiales permite a las larvas cambiar de respiración cutánea a respiración branquial, lo cual resulta en mejor aprovechamiento del oxígeno disponible y en el aumento en la actividad de natación (Gisbert et al. 2002). De esta forma, Rombough (1988) señala que las branquias se forman en una fase tardía durante el desarrollo de las larvas, y mientras no aparezcan las branquias, el consumo de oxígeno está restringido a un intercambio de gases a través de la piel por difusión. Posteriormente, dado que el área-superficie de la piel se expande a una tasa proporcional a $\mathrm{B}^{2 / 3}(\mathrm{~B}=$ biomasa $)$, mientras la demanda por $\mathrm{O}_{2}$ se expande a una tasa proporcional a la $\mathrm{B}^{1,0}$, entonces conforme el organismo crece la respiración cutánea se vuelve limitante (Rombough 2007). De acuerdo con Krogh (1941), la solución es desarrollar estructuras branquiales que por su morfología no están sujetas a las mismas limitaciones geométricas como la piel. La opinión de que las branquias se desarrollan para el intercambio de gases, es una hipótesis que todavía permanece y es la explicación aceptada del porque los peces desarrollan branquias, aunque también se ha sugerido que las branquias se desarrollan para satisfacer la demanda de iones (Li et al. 1995).

La tasa respiratoria en términos de biomasa $\left(\mu \mathrm{LO}_{2} \mathrm{mg}^{-1} \mathrm{Bs}\right.$ $\mathrm{h}^{-1}$ ) disminuye linealmente (datos transformados a logaritmos de base 10) con el incremento en la biomasa de los organismos en general (Savage et al. 2004, West \& Brown 2005, Anderson-Teixeira et al. 2009) y de los peces en particular (Barrionuevo \& Burggren 1999, Feeley et al. 2007, Pelster 2008, Yagi \& Oikawa 2014), siguiendo el modelo potencial $\left(\mathrm{y}=\mathrm{a}^{*} \mathrm{Bs}^{-\mathrm{b}}, \mathrm{y}\right.$ : tasa respiratoria, Bs: biomasa seca, a y b: parámetros de la ecuación). El valor de b en la ecuación potencial generalmente se encuentra entre - 0,2 y - 0,25 (Brett \& Groves 1979, Steinhausen et al. 2005), y aunque hay ligeras variaciones entre especies, los valores se mantienen (Smith \& Brown 1983, West \& Brown 2005, Clark \& Seymour 2009). El valor obtenido en este trabajo fue de $-0,17$, un valor muy cercano al intervalo predicho. Como se ha demostrado en los peces (Bokma 2004, Burggren \& Blank 2009, Hunt von Herbing 2005), la relación alométrica de la tasa de consumo de oxígeno por individuo $\left(\mu \mathrm{LO}_{2}\right.$ larva $\left.^{-1} \mathrm{~h}^{-1}\right)$ con la biomasa corporal del pargo flamenco (datos transformados a logaritmos de base 10) sigue el modelo potencial estándar $\mathrm{y}=\mathrm{a}^{*} \mathrm{Bs}^{\mathrm{b}}, \mathrm{y}$ : tasa metabólica por individuo, a: coeficiente, BS: biomasa corporal y b (la pendiente): exponente de escalamiento. Con base en la biomasa seca, el exponente de escala determinado para el pargo flamenco en este estudio $(0,83)$ fue igual al mencionado por Yagi \& Oikawa (2014) para P. olivaceus y bastante cercano al exponente de escala de 0,79 determinado por Clarke \& Johnston (1999), en su estudio sobre la tasa metabólica de 69 especies de teleósteos. 
Los datos presentados en este trabajo demostraron que el escalamiento del metabolismo con el tamaño corporal de las larvas pargo flamenco, no es substancialmente diferente al reportado para otros teleósteos. Los datos obtenidos son importantes para estimar la demanda de oxígeno durante el cultivo larvario del pargo flamenco. Los resultados obtenidos, combinados con las pérdidas de nitrógeno, pueden ser usados para estimar los requerimientos de alimentación con base en las pérdidas energéticas.

\section{Agradecimientos}

Los autores agradecen a la MPA N García-Aguilar por proporcionar los huevos de pargo flamenco, a J Huerta y A Ibarra por su asistencia técnica y a VWilliams por la traducción del resumen y revisión del texto.

\section{LITERATURA CITADA}

Abdo de la Parra MI \& LE Rodríguez-Ibarra. 2011. Cultivo larvario y requerimientos nutricionales del pargo flamenco, 64 pp. Editorial Académica Española, Berlín.

Abdo-de la Parra MI, LE Rodríguez-Ibarra, F CampilloMartínez, G Velasco-Blanco, N García-Aguilar, LS Álvarez-Lajonchère \& D Voltolina. 2010. Efecto de la densidad de siembra sobre el crecimiento y supervivencia larval del pargo lunarejo Lutjanus guttatus. Revista de Biología Marina y Oceanografía 45: 141-146.

Allen GR. 1995. Lutjanidae. Pargos. En: Fischer W, K Krupp, W Schneider, C Sommer, KE Carpenter \& VH Niem (eds). Guía FAO para la identificación de especies para los fines de la pesca. Pacifico Centro-Oriental,Volumen III Vertebrados Parte 2: 1231-1244. FAO, Roma.

Alvarez-Lajonchère L \& AC Puello-Cruz. 2011. El pargo flamenco: Lutjanus guttatus. Producción controlada de huevos, larvas y juveniles, 169 pp. Clave Editorial, México.

Alvarez-Lajonchère LS, MI Abdo-de la Parra, LE Rodríguez-Ibarra, G Velasco Blanco, A Puello-Cruz, B González-Rodríguez, A Ibarra-Soto \& L Ibarra-Castro. 2012. The scale-up of Spotted Rose Snapper, Lutjanus guttatus, larval rearing at Mazatlan, Mexico. Journal of the World Aquaculture Society 43(3): 411-422.

Ameer Hamsa KM \& MN Kutty. 1972. Oxygen consumption in relation to spontaneous activity and ambient oxygen in five marine teleosts. Indian Journal of Fisheries 12: 76-85.

Anderson-Teixeira KJ, VM Savage, AP Allen \& JF Gillooly. 2009. Allometry and metabolic scaling in ecology. In: Encyclopedia of Life Sciences, 10 pp. John Wiley \& Sons, Chichester. <doi: 10.1002/9780470015902.a0021222>
Aristizabal EO. 2006. Consumo de oxígeno, crecimiento y utilización del alimento durante el desarrollo larval del besugo Pagrus pagrus (Linnaeus, 1758) (Pisces: Sparidae). Revista de Biología Marina y Oceanografía 41: 209-220.

Avilés-Quevedo A, JM Mazón-Suástegui \& F CastellóOrvay. 2008. Avances en el cultivo del pargo flamenco, Lutjanus guttatus un ejemplo a seguir de los pescadores de Bahía Concepción, en Baja California Sur. Industria Acuícola 4: 4-7.

Barrionuevo WR \& WW Burggren. 1999. Oxygen consumption and heart rate in developing zebrafish (Danio rerio): influence of temperature and ambient oxygen. American Journal of Physiology 276: 505-513.

Barrionuevo WR, MN Fernandes \& O Rocha. 2010. Aerobic and anaerobic metabolism for the zebrafish, Danio rerio, reared under normoxic and hypoxic conditions and exposed to acute hypoxia during development. Brazilian Journal of Biology 70(2): 425-434.

Benetti D, C Acosta \& JC Ayala. 1995. Cage and pond aquaculture of marine finfish in Ecuador. World Aquaculture 26: 7-13.

Bokma F. 2004. Evidence against universal metabolic allometry. Functional Ecology 18: 184-187.

Boza-Abarca J, C Calvo-Vargas, N Solis-Ortiz \& J Komen. 2008. Desove inducido y crecimiento del pargo manchado Lutjanus guttatus, en la Estación de Biología Marina de Puntarenas, Costa Rica. Ciencias Marinas 34: 239-252.

Brett JR \& TD Groves. 1979. Physiological energetic. In: Hoar WS, DJ Randall \& JR Brett (eds). Bioenergetics and growth, Fish Physiology 8: 279-352, Academic Press, New York.

Burggren W \& T Blank. 2009. Physiological study of larval fishes: challenges and opportunities. In: Clemmesen C, AM Malzahn, MA Peck \& D Schnack (eds). Advances in early life history study of fish. Scientia Marina 73(S1): 99-110.

Calderer-Reig A. 2001. Influencia de la temperatura y la salinidad sobre el Crecimiento y consumo de oxígeno de la dorada (Sparus aurata L.). Tesis Doctoral, Departamento de Biología Animal, Universitat de Barcelona, Barcelona, $195 \mathrm{pp}$.

Clark TD \& RS Seymour. 2009. Cardiorespiratory physiology and swimming energetics of a high-energy-demand teleost, the yellowtail kingfish (Seriola lalandi). The Journal of Experimental Biology 209: 3940-3951.

Clarke A \& NM Johnston. 1999. Scaling of metabolic rate with body mass and temperature in teleost fish. Journal of Animal Ecology 68: 893-905. 
De la Gándara F. 2003. Efecto de diversos factores sobre el consumo de oxígeno de juveniles de seriola (Seriola dumerili Risso, 1810) en condiciones de cultivo. Tesis Doctoral, Facultad de Biología, Universidad de Murcia, Murcia, 249 pp.

Djawad MI, K Namba, J Matsuura \& K Uematsu. 1995. Oxygen consumption of ayu larvae in fasting condition. Journal of the Faculty of Applied Biological Science 35: 149161.

Feeley MW, DD Benetti \& JS Ault. 2007. Elevated oxygen uptake and high rates of nitrogen excretion in early life stages of the cobia Rachycentron canadum (L.), a fast-growing subtropical fish. Journal of Fish Biology 71: 1662-1678.

Fischer W, F Krupp \& W Schneider. 1995. Guía FAO: Para la identificación de especies para los fines de la pesca. Pacifico Centro-Oriental 3: 1201-1813. FAO, Roma.

García-Ortega A, I Abdo, N Duncan, E Rodríguez, G Velasco, B González, A Puello \& I Marínez. 2005. Larval rearing of rose spotted snapper Lutjanus guttatus under experimental conditions. In: Hendry CI, G van Stappen, M Wille \& P Sorgeloos (eds). Larvi'05- Fish and Shellfish Larviculture Symposium, Special Publication 36: 172-175. European Aquaculture Society, Oostende.

Gisbert E, G Merino, JB Muguet, D Bush, RH Piedrahita \& DE Conklin. 2002. Morphological development and allometric growth patterns in hatchery-reared California halibut larvae. Journal of Fish Biology 61: 1217-1229.

Houde ED \& RC Sckeckter. 1983. Oxygen uptake and comparative energetics among eggs and larvae of the subtropical marine fishes. Marine Biology 72: 283-293.

Houde ED \& CE Zastrow. 1993. Ecosystem- and taxonspecific dynamic and energetics properties of larval fish assemblages. Bulletin of Marine Science 53: 290-335.

Hunt von Herbing I. 2005. The physiological basis for metabolic scaling in animals: a developing perspective. In: Warburton SJ, W Burggren, B Pelster, C Reiber \& J Spicer (eds). Comparative developmental physiology, pp. 83-98. Oxford University Press, New York.

Ibarra-Castro L, L Alvarez-Lajonchère, $\mathbf{N}$ García-Aguilar MI Abdo-de la Parra \& LE Rodríguez-Ibarra. 2012. Generation cycle closure of the spotted rose snapper, Lutjanus guttatus, in captivity. Revista de Biología Marina y Oceanografía 47(2): 333-337.

Idrisi N, T Capo, S Luthy \& J Seraphy. 2002. Behaviour, oxygen consumption and survival of stressed juvenile sailfish (Istiophorus platypterus) in captivity. Marine and Freshwater Behaviour and Physiology 36: 51-57.

Ishibashi Y, K Inoue, H Nakatsukasa, Y Ishitani, S Miyashita \& O Murata. 2005. Ontogeny of tolerance to hypoxia and oxygen consumption of larval and juvenile red sea bream, Pagrus major. Aquaculture 244: 331-340.
Iwai T \& GM Hughes. 1977. Preliminary morphometric study on gill development in Black sea bream (Acanthopagrus schlegeli). Bulletin of the Japanese Society 43: 929-934.

Jobling M. 1994. Respiration and metabolism. In: Jobling M (ed). Fish bioenergetics, pp. 121-146. Chapman \& Hall, London.

Klimant I, V Meyer \& M Kühl. 1995. Fiber-optic oxygen micro sensors, a new tool in aquatic biology. Limnology and Oceanography 40: 1159-1165.

Krogh A. 1941. Comparative physiology of respiratory mechanisms, 172 pp. University of Pennsylvania Press, Philadelphia.

Li J, J Eygensteyn, R Lock, P Verbost, A Heijden, S Bonga \& G Flik. 1995. Branchial chloride cells in larvae and juveniles of freshwater tilapia Oreochromis mossambicus. Journal of Experimental Biology 198: 2177-2184.

Miyashima A, T Kotani, H Tawa \& H Fushimi. 2012. Relationship between oxygen consumption, growth and survival of larval fish. Aquaculture Research 43: 679-687.

Oikawa S, M Takemori \& Y Itazawa. 1992. Relative growth of organs and parts of a marine teleost, the porgy, Pagrus major, with special reference to metabolism-size relationship. Japanese Journal of Ichthyology 39: 243-249.

Oozeki Y \& R Hirano. 1994. Changes in oxygen consumption rate during development of larval japanese whiting, Sillago japonica. Japanese Journal of Ichthyology 41: 207-214.

Pelster B. 2008. Gas exchange. In: Finn RN, BG Kapoor \& NH Enfield (eds). Fish larval physiology, pp. 91-117 pp. Science Publishers, Enfield.

Rombough PJ. 1988. Respiratory gas exchange, aerobic metabolism, and effects of hypoxia during early life. In: Hoar WS \& DJ Randall (eds). Fish physiology, Vol. 11. The physiology of developing fish: Part A: Eggs and larvae, pp 59-114. Academic Press, San Diego.

Rombough PJ. 2007. Oxygen as a constraining factor in egg size evolution in salmonids. Canadian Journal of Fisheries and Aquatic Sciences 64: 692-699.

Savage VM, JF Gillooly, WH Woodruff, GB West, AP Allen, BJ Enquist \& JH Brown. 2004. The predominance of quarter power scaling in biology. Functional Ecology 18: 257 282.

Smith KL \& NO Brown. 1983. Oxygen consumption of pelagic juveniles and demersal adults of the deep-sea fish Sebastolobus altivelis, measured at depth. Marine Biology 76:325-332.

Steinhausen MF, JF Steffensen \& NG Andersen. 2005. Tail beat frequency as a predictor of swimming speed and oxygen consumption of saithe (Pollachius virens) and whiting (Merlangius merlangus) during forced swimming. Marine Biology 148: 197-204. 
Torres JJ, RI Brightman, J Donell \& J Harvey. 1996. Energetics of larval red drum, Sciaenops ocellatus. Part 1: oxygen consumption, specific dynamic action, and nitrogen excretion. Fishery Bulletin 94: 756-765.

West GB \& JH Brown. 2005. The origin of allometric scaling laws in biology from genomes to ecosystems: towards a quantitative unifying theory of biological structure and organization. The Journal of Experimental Biology 208: 15751592.
Winberg GG. 1960. Rate of metabolism and food requirements of fishes. In: Fry FEJ \& WE Ricker (eds). Fisheries Research Board of Canada, Translation Series 194: 1-21. Biological Station, Nanaimo.

Yagi M \& S Oikawa. 2014. Ontogenetic phase shifts in metabolism in a flounder Paralichthys olivaceus. Scientific Reports 4,7135. <doi:10.1038/srep07135>

Zar JH. 1999. Biostatistical analysis, 660 pp. Prentice Hall, Upper Saddle River.

Recibido el 21 de mayo de 2015 y aceptado el 16 de diciembre de 2015

Editor: Claudia Bustos D. 